\title{
"Pour une Prospective du Travail. Les mutations et transitions du Travail à hauteur d'Hommes" [Résumé]
}

Valérie Pueyo

\section{CpenEdition}

Journals

Édition électronique

URL : http://journals.openedition.org/activites/6349

DOI : 10.4000 /activites.6349

ISSN : 1765-2723

Éditeur

ARPACT - Association Recherches et Pratiques sur les ACTivités

Référence électronique

Valérie Pueyo, " "Pour une Prospective du Travail. Les mutations et transitions du Travail à hauteur d'Hommes" [Résumé] ", Activités [En ligne], 18-1 | 2021, mis en ligne le 15 avril 2021, consulté le 17 avril 2021. URL : http://journals.openedition.org/activites/6349; DOI : https://doi.org/10.4000/ activites.6349

Ce document a été généré automatiquement le 17 avril 2021.

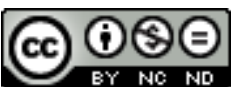

Activités est mis à disposition selon les termes de la licence Creative Commons Attribution - Pas d'Utilisation Commerciale - Pas de Modification 4.0 International. 


\title{
"Pour une Prospective du Travail. Les mutations et transitions du Travail à hauteur d'Hommes" [Résumé]
}

\author{
Valérie Pueyo
}

\section{RÉFÉRENCE}

Valérie Pueyo, Pour une Prospective du Travail. Les mutations et transitions du Travail à hauteur d'Hommes. Habilitation à Diriger des recherches - Ergonomie, École Doctorale 483 ScSo, Université Lumière Lyon 2. Soutenance le 24 janvier 2020

\section{NOTE DE L'ÉDITEUR}

\section{Jury}

- Pascal BEGUIN, Garant, Professeur Université Lumière Lyon 2

- Benoit DEDIEU, Examinateur, Directeur de Recherche INA-SAD

- Viviane FOLCHER, Rapporteure, MCF-HDR Université Paris 8

- Alain GARRIGOU, Rapporteur, Professeur Université de Bordeaux

- Laerte SZNELWAR, Rapporteur, Professeur Université de São Paulo

- Pierre VELTZ, Examinateur, Professeur Emérite École des Ponts Paris Tech

1 Dès les années 70, Alain Wisner avait engagé l'Ergonomie dans ce qu'il appelait alors "la bataille du travail réel». Mais depuis cette période, le "grand récit industriel» (Lyotard, 1979) qui tissait les liens entre projets politique, technique et sociétal s'est effondré. Les modèles productifs (Boyer, \& Freyssenet, 2000) actuels témoignent d'une "modernisation sans modernité» (Stiegler, 2011) menée à un rythme effréné entre promesses enchanteresses et nécessités. La mécanique des changements, lancée à plein 
régime, s'accompagne d'une absence de sens et d'un oubli de ce qui fait l'Humain (Ricoeur, 1967). Et les trajectoires évolutives des milieux productifs et des populations au travail sont de moins en moins articulées. Ce découplage âprement vécu détruit l'idée de progrès entendu comme projet sociétal; et il sape l'ambition d'un futur souhaitable ou simplement meilleur.

2 Loin de se résigner à ce fatalisme, l'objectif de ce document est de rendre compte d'une trajectoire de recherche qui s'est alors interrogée sur les possibilités de développement d'une prospective du travail, dont la finalité serait de chercher les voies « pour un demain qui reste à inventer, qui dépendra de nous " (Berger, 1964). Dans la mesure où il n'est pas possible de s'engager dans une telle démarche sans prendre en compte les projets que les protagonistes forment pour eux-mêmes, l'ambition est de rechercher, dans l'expérience des travailleurs, les ressources sur lesquelles il est possible de s'appuyer pour former, expérimenter et accompagner, des alternatives souhaitables au présent.

3 Le premier chapitre discute l'analyse qu'il est possible de faire des dynamiques de découplage entre les milieux productifs et les populations, en évolutions. Cette analyse dénaturalisante est conduite au prisme du vieillissement (alors appréhendé comme un analyseur et non comme une ontologie). Sous cet angle, le découplage peut être examiné selon trois modèles. Le premier modèle se centre sur les altérations que provoquent les systèmes de travail sur les populations. Le second modèle met en évidence les voies qu'empruntent les salariés pour se préserver grâce à l'expérience. On propose un troisième modèle qui vise à appréhender l'élan vital de conformation des protagonistes concernés par les changements et ce qu'ils cherchent à y construire. Ce troisième modèle, dit de la Construction, prend appui sur l'unité d'analyse de l'ergonomie - l'activité -. Mais il oblige à des changements d'unité conséquents : aux plans temporels, en termes de périmètres, de niveaux, et enfin de domaines d'appréhension.

4 De ce fait, le second chapitre indique ce que le modèle de la Construction retient : i) du tournant développemental de l'Ergonomie pour saisir les dynamiques et changements en jeu au plan de l'activité, ii) des Sciences de la Gestion pour les appréhender au plan des structures, et iii) de l'Histoire pour les penser au plan des sociétés, des communautés et des temporalités. Ces trois plans d'analyse en interaction définissent les éléments retenus pour mieux appréhender l'architecture fonctionnelle des changements en train de se faire - les transitions - et leur cristallisation - les mutations. Au plan de l'activité, ce sont les rapports au monde, au plan des structures, ce sont les systèmes hétérodéterminants, au plan de la société, des arrière-plans structurants. Enfin, on pose que cette architecture peut être décomposée et recomposée dans une dynamique que l'on qualifie de "marche des morphogenèses ".

Le troisième chapitre rend compte d'un premier effort d'analyse des mutations et des transitions du travail, à partir "d'indicateurs démographiques des changements" construits pour l'occasion. Et il fait un bilan de ce que leur usage nous enseigne. On présente quatre familles d'indicateurs élaborées à partir d'outils démographiques que sont: les structures d'âge, les effets d'âge, les effets de période et les effets de génération. Ces indicateurs valident la pertinence de l'architecture fonctionnelle des changements. Ils précisent également ce que sont les facteurs de mutations et de transitions, à quels plans ils se situent, et leur variété temporelle. Mais simultanément, leur usage nous place devant à une énigme : ils détectent les traces d'une constellation 
composée par les Humains, qui organise en cohérence les plans et les éléments de l'architecture fonctionnelle. Cependant, ils ne permettent pas d'en saisir la nature.

6 Le quatrième chapitre assume alors un changement de perspective qui consiste à donner la part belle à ces élans de composition. On s'y interroge sur la nature de cette constellation constituée par les protagonistes et qui semble opérer comme un espace d'ordre. L'enjeu est alors d'appréhender cet espace d'ordre en trame (ses dimensions structurantes) et en substance (son sens) au plan des concepts; et de construire des indicateurs pour en faire l'analyse dans des situations concrètes. On s'appuie sur Mannheim et sur Dewey pour examiner comment les Hommes se mettent en mouvement pour construire ces espaces d'ordre que l'on identifie plus précisément comme des entéléchies structurées, à l'échelle des générations. Et puisque cette mise en mouvement renvoie au vécu des protagonistes, on s'appuie sur Ricoeur pour comprendre ce que les récits des travailleurs, les événements qu'ils évoquent, et les œuvres qu'ils commémorent peuvent nous apprendre sur ces entéléchies ainsi appréhendées au prisme de l'expérience des générations.

7 Le cinquième chapitre rend compte de ce que révèle un tel cadre d'analyse lorsqu'on le met en œuvre. Il met en évidence des compositions qui peuvent aller au-delà d'entéléchies structurées à l'aune d'une génération. En s'appuyant sur un cas, on montre que ces constructions relèvent plutôt d'une utopie concrète au sens de Bloch (1976), qui s'exprime et se cristallise dans un dessein, puis dans un projet-chantier supporté par un contrat de base. Le contrat de base doit être entendu comme "un dispositif industrieux expérientiel » qui témoigne de la volonté humaine d'être acteur du changement et la soutient. Tandis que le projet-chantier rend compte de la dimension historicisée, située et contingente de l'expression d'une utopie concrète. À l'examen, contrat de base et projet-chantier sont normativement pertinents pour penser et conduire des mutations et transitions souhaitables et souhaitées, dans la mesure où ils s'avèrent favorables pour le Travail et la Santé.

8 La conclusion se veut ouverte sur le futur. Elle capitalise les acquis de ce parcours pour proposer une démarche pour former des " projets chantiers " étayés par des « contrats de base", mais en tenant compte d'une diversité de situations. Elle annonce un programme dont la finalité est de contribuer à la mise en œuvre d'une prospective $d u$ travail humaniste indisciplinée au service de projets relevant d'utopies concrètes. Ce programme proposé pour lutter et agir contre une pensée et un avenir qui se portent sombres et résignés (Lecourt, 1997) constitue un changement paradigmatique. Il engage non plus uniquement à adapter le travail à l'Humain en s'inscrivant dans le régime de travail actuel, mais à participer à la conception d'un travail souhaitable, profondément humain dans un monde où les défis environnementaux et sociétaux sont multiples. Cela oblige en particulier à mener un débat sur la façon dont la communauté des ergonomes assume ou pas, collectivement, la portée politique du travail, du point de vue du vivre ensemble, de sa gouvernance. Cela implique de discuter de ses pratiques en responsabilités. Responsabilités d'autant plus grandes qu'elles accompagnent l'espérance d'utopies concrètes. Une espérance alerte qui n'est pas un espoir insensé demandant toujours plus de promesses, mais qui oblige à l'action, à la confrontation, à l'épreuve et au débat. Une espérance tendue par la conscience d'un au-delà de l'existant lié à une nécessité commune. Une conscience prête à s'attaquer aux problèmes dont les Humains irrésignés ne savent pas s'ils pourront les résoudre. Mais dont ils savent, pour 
reprendre Gaston Berger (1964), qu'il ne s'agit pas uniquement de les résoudre, mais de les poser comme des projets à réaliser si l'on veut.

\section{BIBLIOGRAPHIE}

Berger, G. (1964). Phénoménologie du temps et prospective. Paris : PUF.

Bloch, E. (1976). Le principe espérance, tome I. Paris : Gallimard.

Boyer, R., \& Freyssenet, M. (2000). Les modèles productifs. Paris : La Découverte (Coll. Repères).

Lecourt, D. (1997). L'Avenir du progrès. Paris: Éditions Textuel.

Lyotard, J-F. (1979). La condition post-moderne. Rapport sur le savoir. Paris: Éditions de Minuit.

Ricoeur, P. (1967). Plaidoyer pour l'utopie ecclésiale. Labor et Fides.

Stiegler, B. (2011). Régressions et progressions vers l'ultramodernité. Les entretiens de l'Institut Diderot, l'avenir du progrès.

\section{AUTEURS}

\section{VALÉRIE PUEYO}

IETL, Lyon 2, EVS UMR 5600, Labex IMU 